\title{
diffusion-î̉urdarrentals
}

The Open-Access Journal for the Basic Principles of Diffusion Theory, Experiment and Application

\section{Transient Behavior of a Zeolite Membrane under non-Linear Conditions}

\author{
Douglas M. Ruthven \\ Department of Chemical and Biochemical Engineering \\ University of Maine, \\ Orono, ME, 04469, USA
}

\begin{abstract}
A simplified mathematical model is proposed to provide an analytic solution for the transient behavior of a Langmuirian zeolite membrane subjected to a step increase in sorbate pressure at the upstream surface at time zero. The concentration profiles derived from the simplified model agree with those calculated by numerical solution of the governing partial differential equation. The model is used to investigate the validity of the classical time-delay method of measuring diffusion in the adsorbed phase. Under non-linear conditions the time delay depends on the degree of non-linearity as well as on the diffusional time constant. Under highly non-linear conditions application of the classical linear model yields apparent diffusivity values that are about four times larger than the limiting value $\left(D_{o}\right)$.

The new model is used to reanalyze experimental permeation diffusivity data for $\mathrm{p}$ xylene in a single crystal zeolite membrane. The resulting Do values are shown to be consistent with the values determined by other experimental techniques.

The transient behavior of a membrane, subjected to a step change in the sorbate partial pressure on the upstream side is a classic problem in diffusion theory ${ }^{(1)}$, and provides the basis for a well established experimental technique for measuring the diffusion coefficient ${ }^{(2)}$. The existing theory is, however, limited to linear systems with a constant diffusivity. These conditions are often reasonably closely fulfilled for the diffusion of light gases in polymeric membranes but for heavier species and especially for zeolite membranes in which the equilibrium isotherm is generally non-linear, the assumption of a linear system with constant diffusivity is clearly invalid. Nevertheless the linear theory has frequently been applied to such systems ${ }^{(3-5)}$. It therefore seems important to re-visit the basic analysis of membrane behavior under transient conditions in order to develop a more appropriate model for non-linear systems.
\end{abstract}

\section{Linear Systems}

In order to understand the behavior of non-linear systems it is helpful first to review briefly the classical linear theory. We consider a membrane of thickness $(\ell)$ subjected at time zero to a step increase in the sorbate concentration (adsorbed, absorbed or dissolved) to $c_{1}$ at the upstream face while the permeate face is maintained at a very low sorbate concentration (essentially zero) either by pulling a vacuum or by purging at a sufficiently high rate with an inert sweep gas. For a constant diffusivity system the behavior is described by Fick's second equation: 


$$
\frac{\partial c}{\partial t}=D \frac{\partial^{2} c}{\partial x^{2}}
$$

with the initial and boundary conditions:

$$
\begin{array}{lll}
\mathrm{t}=\mathrm{o}: & \mathrm{o}>\mathrm{x}>\ell, & \mathrm{c}=\mathrm{f}(\mathrm{x}) \\
\mathrm{t}>\mathrm{o}: & \mathrm{x}=\mathrm{o}, & \mathrm{c}=\mathrm{c}_{1} \\
& \mathrm{x}=\ell, & \mathrm{c}=\mathrm{o}
\end{array}
$$

The solution is given by ${ }^{(1)}$ :

$$
C=\frac{c}{c_{1}}=1-X-2 \sum_{n=1}^{\infty} \frac{\sin (n \pi X)}{n \pi} \cdot e^{-n^{2} \pi^{2} \tau}+2 \frac{c_{0} \sin (n \pi x)}{c_{1}} e^{-n^{2} \pi^{2} \tau} I(n)
$$

where $\mathrm{X}=\mathrm{x} / \ell, \tau=\mathrm{Dt} / \ell^{2}$ and $I(n)=\int_{X^{1}=0}^{1.0} f\left(X^{1}\right) \cdot \sin \left(n \pi X^{1}\right) d X^{1}$.

When the initial loading is zero $(f(X)=0), I(n)=0$ and the final term of Eq. 3 vanishes. In the usual experimental system the initial loading is zero and the total quantity of sorbate passing through the membrane $Q\left[=\int_{0}^{t} J d t\right]$ is measured as a function of time.

From Eq. 3:

$$
-\left[\frac{\partial c}{\partial X}\right]_{X=1}=1+2 \sum_{n=1}^{\infty}(-1)^{n} e^{-n^{2} \pi^{2} \tau}
$$

whence:

$$
\frac{Q}{\ell c_{1}}=\int_{\tau^{1}=0}^{\tau}\left[-\frac{\partial C}{\partial X}\right]_{X=1} . d \tau^{1}=\tau-\frac{1}{6}-\frac{2}{\pi^{2}} \sum_{n=1}^{\infty} \frac{(-1)^{n} e^{-n^{2} \pi^{2} \tau}}{n^{2}}
$$

At long times this reduces to:

$$
\frac{Q}{\ell c_{1}}=\tau-\frac{1}{6}+\frac{2}{\pi^{2}} e^{-\pi^{2} \tau}
$$

and eventually to the steady state:

$$
\frac{Q}{\ell c_{1}}=\tau-\frac{1}{6}
$$


Measurement of the time delay $\left(t_{d}\right)$ by extrapolation of this asymptote thus yields directly the diffusional time constant since:

$$
\tau_{d}=\frac{D t_{d}}{\ell^{2}}=\frac{1}{6}
$$

The advantage of this approach is that knowledge of the solubility (or the Henry constant) is not required. Under steady state conditions:

$$
\frac{1}{\ell c_{1}} \cdot \frac{d Q}{d \tau}=1.0 ; \quad J=\frac{d Q}{d t}=\frac{D c_{1}}{\ell}=\frac{K D p_{1}}{\ell}
$$

Thus, if both the upstream pressure $\left(p_{l}\right)$ and the membrane thickness are known, both the diffusivity and the Henry constant can be determined from the same experiment.

The form of the transient profiles and the flow through the membrane, calculated from Eqs. 3 and 5, are shown in figure 1. At short times such that the distance penetrated by the concentration front is less than the membrane thickness the system is equivalent to diffusion into a semi-infinite medium. The transient concentration profile is then given by:

$$
C=\operatorname{erfc}(y)
$$

where $y=x / 2 \sqrt{D t}=X / 2 \sqrt{\tau}$. At the breakthrough time $\left(\tau_{0} \approx 0.0625\right)$ the profiles calculated from Eqs. 3 and 10 are identical, as may be seen from figure 1(a).

\section{Non-Linear Systems}

\section{$\underline{\text { Steady State }}$}

The assumption of a constant (concentration independent) diffusivity is inappropriate for non-linear systems. The flux is determined by the gradient of chemical potential, rather than by the concentration gradient,

$$
J=-B c \frac{\partial \mu}{\partial x}
$$

If the equilibrium relationship is of Langmuir form:

$$
\theta=\frac{c^{*}}{c_{s}}=\frac{b p}{1+b p}
$$




\section{Transient Concentration Profiles (Linear System)}

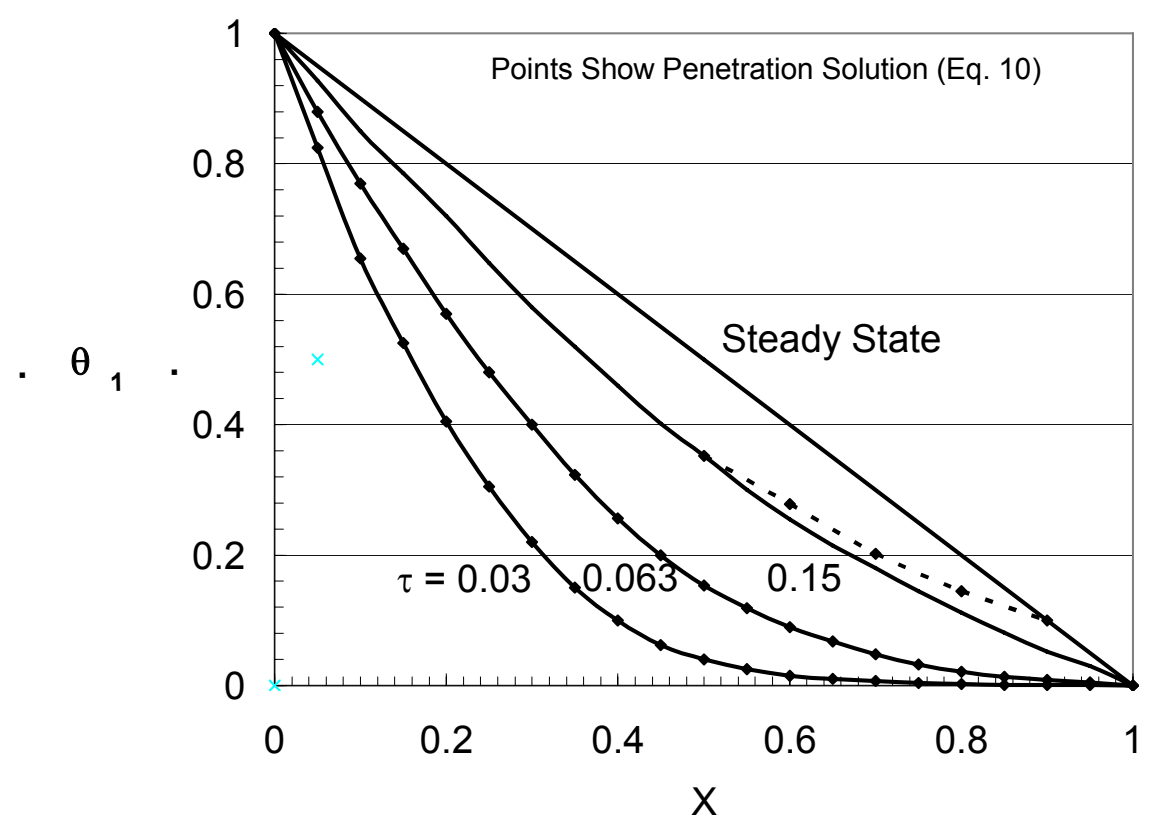

Fig. 1a

Flow through Membrane

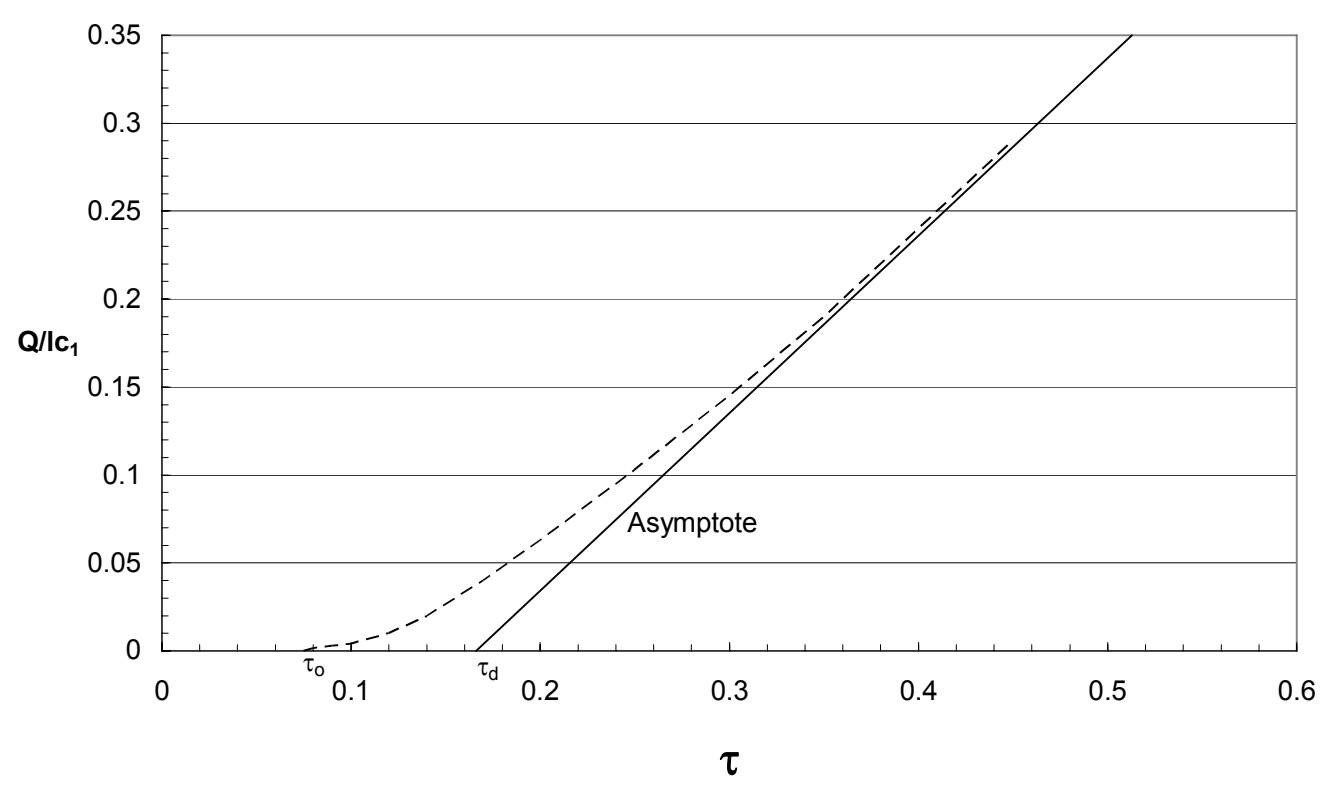

Fig.1b

Fig. 1 (a) Transient concentration profiles through the membrane for a linear system showing the approach to steady state and the comparison between the penetration solution (Eq. 10) and the exact solution (Eq. 3).

(b) Flow through the membrane $\left(Q / \ell c_{1}\right)$ as a function of time showing the approach to the steady state. Breakthrough and delay times are indicated. 
Eq. 11 reduces to:

$$
J=-B R T\left(\frac{c^{*}}{p}\right) \frac{\partial p}{\partial x}=-\frac{D_{0} b c_{s}}{1+b p} \cdot \frac{\partial p}{\partial x}
$$

Integrating across the membrane (from $x=0, c=c_{1}$ ) yields:

$$
\frac{J x}{D_{0} c_{s}}=\ln \left(\frac{1+b p_{1}}{1+b p}\right)=\ln \left(\frac{1-\theta}{1-\theta_{1}}\right)
$$

where $\theta=c / c_{s}=\left(c / c_{1}\right) \theta_{1}=C \theta_{1}$ and, at the permeate side $(x=\ell, \theta=0)$ :

$$
\frac{J \ell}{D_{0} c_{s}}=-\ell n\left(1-\theta_{1}\right)
$$

The steady state concentration profile through the membrane is therefore given by:

$$
C=\theta / \theta_{1}=\frac{1-\left(1-\theta_{1}\right)^{(1-X)}}{\theta_{1}}
$$

with the slope:

$$
\frac{d \theta}{d X}=\frac{\theta_{1}}{c_{1}} \frac{d c}{d X}=(1-\theta) \ln \left(1-\theta_{1}\right)
$$

The flow through the membrane under steady state conditions is determined by the concentration gradient at the permeate face and is given by:

$$
\frac{\Delta Q}{\ell c_{1}}=-\left.\int_{\tau_{1}}^{\tau_{2}} \frac{1}{c_{1}} \cdot \frac{d c}{d X}\right|_{X=1} \cdot d \tau=-\frac{\ell n\left(1-\theta_{1}\right)}{\theta_{1}} \cdot \Delta \tau
$$

Comparison with Eq. 9 shows that for a Langmuirian system the steady state flow (i.e. the limiting slope of a plot of $Q / \ell c_{1}$ vs $\left.\tau\right)$ is increased by the factor $-\ell n\left(1-\theta_{1}\right) / \theta_{I}$ relative to the linear case. The apparent diffusivity derived from such a plot will therefore be increased by this same factor.

\section{$\underline{\text { Transient Behavior }}$}

The Langmuirian system is formally equivalent to a Fickian system in which the diffusivity varies with sorbate concentration in accordance with:

$$
D=\frac{D_{0}}{1-\theta}=\frac{D_{0}}{1-\theta_{1} C}
$$


The transient behavior is described by the partial differential equation:

$$
\frac{\partial C}{\partial \tau}=\frac{\partial}{\partial X}\left[\frac{D}{D_{0}} \cdot \frac{\partial C}{\partial X}\right]=\frac{\partial}{\partial X}\left[\frac{1}{\left(1-\theta_{1} C\right)} \cdot \frac{\partial C}{\partial X}\right]
$$

An analytic solution to this equation for the boundary conditions appropriate for diffusion into a semi-infinite medium (Eq. 2 with the final boundary condition replaced by $x \rightarrow \infty, c \rightarrow 0$ ) has been obtained by Fujita ${ }^{(6)}$ and this solution has been summarized by Crank ${ }^{(1)}$. However, Fujita's approach, which uses the Boltzmann transformation $(y=x / 2 \sqrt{D t})$, cannot be extended to the spatially finite boundary conditions appropriate for a membrane of finite thickness (Eq. 2), although the problem can of course be solved numerically for any specified values of the parameters $D_{o}, \theta_{1}$ and $\ell$. A more insightful analytic approximation to the solution can however be obtained by recognizing that Fujita's solution is applicable exactly to the finite membrane up to the breakthrough time, and, if the concentration at the permeate side is maintained close to zero (as in the usual experimental system), the subsequent response, which depends on the concentration gradient at the permeate face, will be amenable to a simple linear analysis;

The steady state concentration profile through the membrane is given by Eq. 17 and the limiting slope (at the permeate face) is therefore given by:

$$
\left.\frac{1}{c_{1}} \cdot \frac{d c}{d X}\right|_{X=1}=\left.\frac{1}{\theta_{1}} \frac{d \theta}{d X}\right|_{X=1}=\frac{\ln \left(1-\theta_{1}\right)}{\theta_{1}}
$$

For a linear system at steady state this slope is constant through the membrane so we consider a section of the membrane (at the permeate side) of thickness $\ell^{l}$ such that:

$$
\frac{\ell^{1}}{\ell}=\frac{\theta_{1}}{-\ln \left(1-\theta_{1}\right)}
$$

We consider distance $\left(x^{l}\right)$ measured from the point $x=\ell-\ell^{l}$ (i.e. $\left.X^{l}=x^{1} / \ell^{l}\right)$ and time $\left(\tau^{l}=D_{0}\right.$ $\left.\tau^{l} / \ell^{12}\right)$ measured from the breakthrough time $\left(\tau_{0}\right)$. At this time the spatial profile $C=f\left(x^{l}, \tau^{l}, \theta_{1}\right)$ which corresponds to the initial profile in the region $0<X^{l}<1$ is given by Fujita's solution. The variation of breakthrough time with $\theta_{1}$ from Fujita's solution, is shown in figure 2. Provided that the boundary condition at $x^{l}=0$ is consistent with the final steady state profile its precise form has only a minor effect on the transient response at the permeate face $\left(x=\ell, x^{I}=\ell^{I}\right)$. We therefore consider the region $0<X^{l}<1$ as equivalent to a linear system in which the response is given by Eq. 3 (with $X$ and $\tau$ replaced by $X^{l}$ and $\tau^{l}$ ) subject to the boundary conditions:

$$
t<0: \quad C=\frac{c_{0}}{c_{1}} f\left(X^{1}\right)
$$

where $f\left(X^{I}\right)$ represents the concentration profile at breakthrough over the region $\left(\ell-\ell^{l}\right) / \ell<X<$ 1.0 from Fujita's solution. 


$$
\begin{aligned}
t>0: & X^{1}=0, & & c=c_{1} \\
X^{1} & =1, & & c=0
\end{aligned}
$$

The solution to this problem therefore reduces to calculating the integral $I(n)$ for Fujita's solution. In principle this integral can be calculated from the analytic expression provided (see the appendix) but the formal solution is complicated and it is easier to approximate the profile by a simple algebraic form. The obvious choice is a negative exponential $\left(c_{0} / c_{1}=e^{-k X^{1}}\right)$ for which:

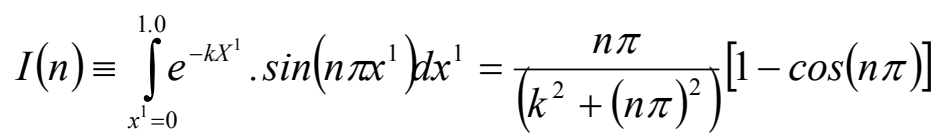

With this approximation the expression for the concentration profile (within the region $0<X^{l}<1.0$ ) and the flow through the membrane (at $\left.X=X^{l}=1.0\right)$ are given by:

$$
\begin{aligned}
& \frac{c}{c_{1}}=1-X^{1}-2 \sum_{n=1}^{\infty} \frac{\sin \left(n \pi X^{1}\right)}{n \pi} \cdot e^{-n^{2} \pi^{2} \tau^{1}}+\frac{2 c_{0}}{c_{1}} \sum_{n=1}^{\infty} \sin \left(n \pi X^{1}\right) \cdot\left(\frac{n \pi}{k^{2}+(n \pi)^{2}}\right) \cdot e^{-n^{2} \pi^{2} \tau^{1}} \\
& -\frac{2 c_{0}}{c_{1}} \sum \sin \left(n \pi X^{1}\right)\left(\frac{n \pi}{k^{2}+(n \pi)^{2}}\right) \cdot e^{-n^{2} \pi^{2} \tau^{1}} \\
& -\left.\frac{1}{c_{1}} \cdot \frac{\partial c}{\partial X^{1}}\right|_{X^{1}=1}=1+2 \sum_{n=1}^{\infty} \cos (n \pi) \cdot e^{-n^{2} \pi^{2} \tau^{1}} \\
& -2 \frac{c_{0}}{c_{1}} \sum_{n=1}^{\infty} \frac{(n \pi)^{2}}{k^{2}+(n \pi)^{2}} \cdot \cos (n \pi) \cdot e^{-n^{2} \pi^{2} \tau^{1}} \\
& +2 \frac{c_{0}}{c_{1}} \sum_{n=1}^{\infty} \frac{(n \pi)^{2}}{k^{2}+(n \pi)^{2}} \cdot \cos ^{2}(n \pi) \cdot e^{-n^{2} \pi^{2} \tau^{1}} \\
& \frac{Q}{\ell^{\prime} c_{1}}=-\left.\int_{0}^{\tau^{1}} \frac{1}{c_{1}} \cdot \frac{\partial c}{\partial X^{1}}\right|_{X^{1}=1} . d \tau=\tau^{1}-\frac{1}{6}+\frac{4 c_{0}}{c_{1}} \sum_{m=0}^{\infty} \frac{1}{k^{2}+(2 m+1)^{2} \pi^{2}} \\
& -2 \sum_{n=1}^{\infty} \frac{(-1)^{n}}{n^{2} \pi^{2}} \cdot e^{-n^{2} \pi^{2} \tau^{1}} \\
& -4 \frac{c_{0}}{c_{1}} \sum_{m=0}^{\infty} \frac{e^{-(2 m+1)^{2} \pi^{2} \tau^{1}}}{k^{2}+(2 m+1)^{2} \pi^{2}}
\end{aligned}
$$

The time delay (defined by the intercept of the long time asymptote) is given by: 


$$
\tau_{d}^{1}=\frac{1}{6}-4 \frac{c_{0}}{c_{1}} \sum_{m=0}^{\infty} \frac{1}{k^{2}+(2 m+1)^{2} \pi^{2}}
$$

The overall time delay is given by:

$$
\tau_{d}=\tau_{0}+\left(\frac{\ell^{1}}{\ell}\right)^{2} \tau_{d}^{1}=\tau_{0}+\left[\frac{\theta_{1}}{\ln \left(1-\theta_{1}\right)}\right]^{2} \tau_{d}^{1}
$$

where $\tau_{0}$ (from Fujita's solution) is obtained from figure 2 and $\tau_{d}^{1}$ from Eq. 28. the variation of $\tau_{d}$ with $\theta_{1}$ is almost linear as shown in figure 2 .

The development of the concentration profile (for $\theta_{1}=0.8$ ) is shown in figure 3 . The profiles calculated by numerical solution of Eq. 20 lie very close to those that would be obtained by interpolation between the Fujita solution (for a semi-infinite medium) and the linear model (for the region $0<X^{1}<1.0,0.503<X<1.0$ ) thus confirming the validity of the approximate model.

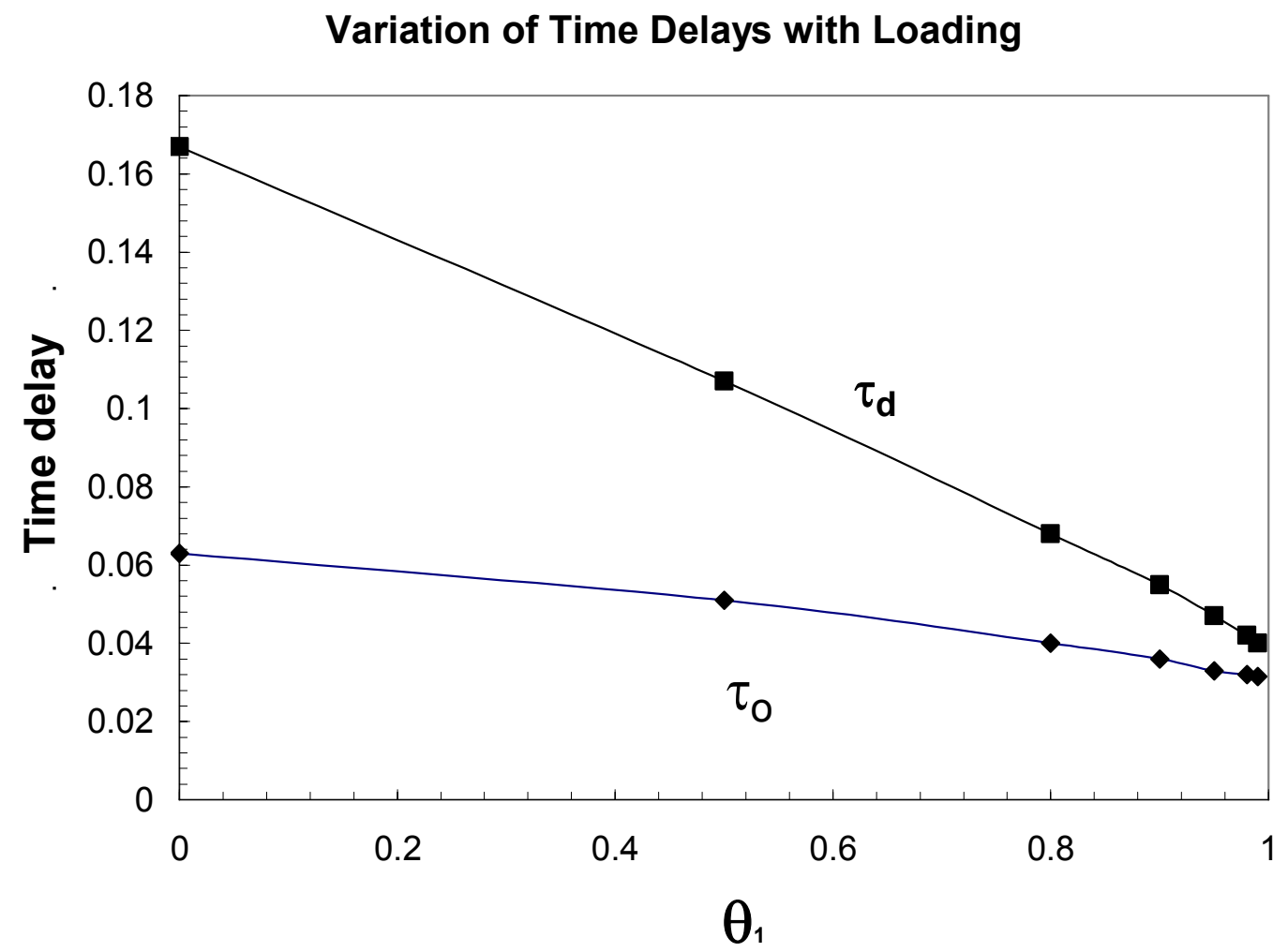

Fig. 2 Variation of breakthrough time $\left(\tau_{0}\right)$ with $\theta_{1}$. Values of $\tau_{0}$ are calculated from Fujita's solution taking $C=0.001$ as the breakpoint. Values of $\tau_{d}$ are calculated from Eqs. 28 and 29. 
Transient Concentration Profiles $\left(\theta_{1}=0.8\right)$

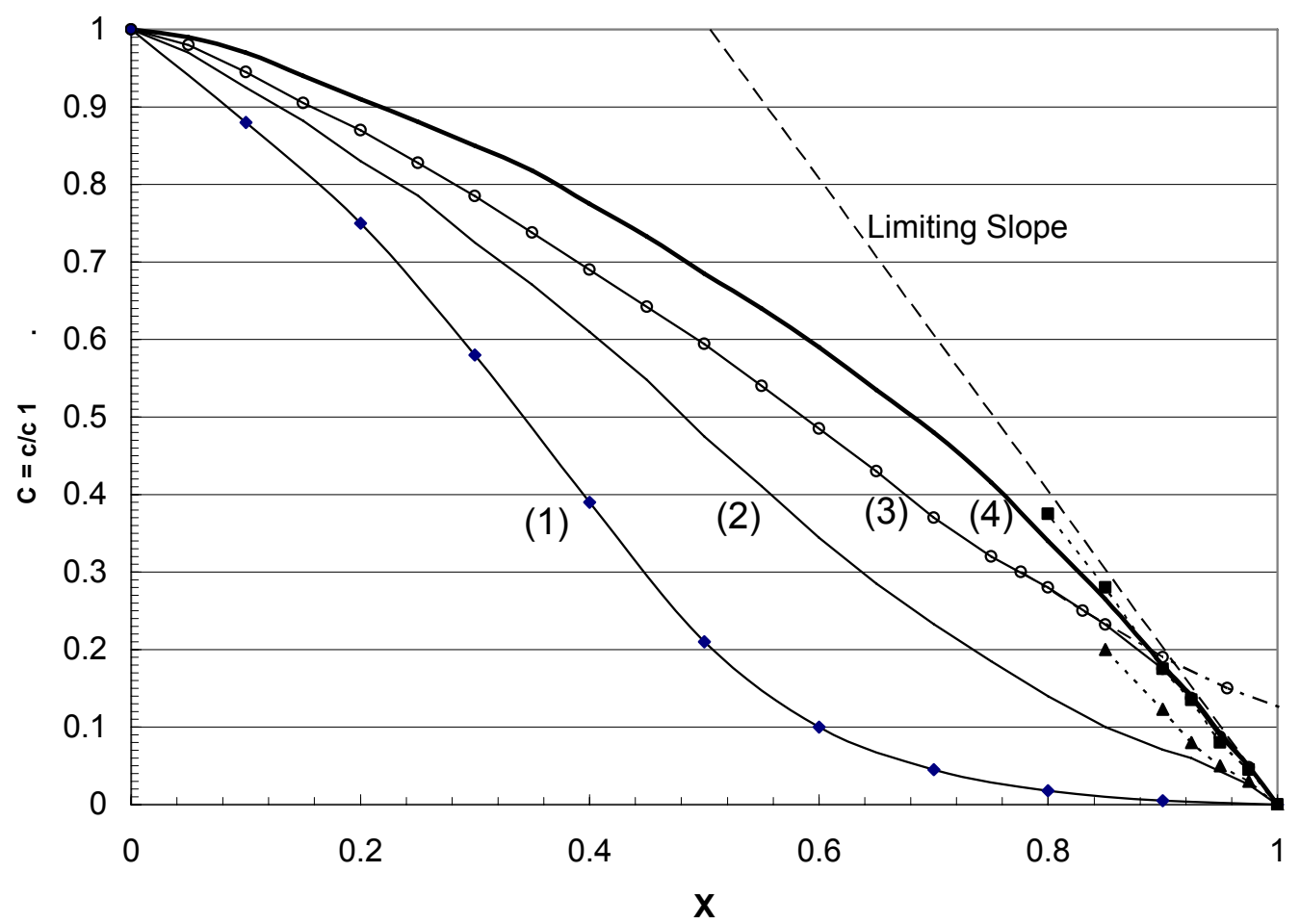

Fig. 3. Development of concentration profile $C(X, \tau)$ for $\theta_{1}=0.8$.

(1) Breakthrough profile $\left(\tau_{0}=0.04\right)$ showing comparison between Fujita's solution ( ) and the numerical solution derived from Eq. 20 ( ). (2,3) Profiles calculated numerically from Eq. 20 for $\tau=0.08$ and 0.12 ( ). Also shown are the limiting profiles for these values of $\tau$ calculated from the simplified model $(\mathbf{\Lambda}, \mathbf{-})$ and the profile from Fujita's solution (o).

(4) Steady state profile calculated from Eq. 16.

Figure 4 shows the transient behavior as a plot of $Q / \ell c_{1}$ vs $\tau$ calculated from Eq. 27 for various values of $\theta_{1}$. With increasing non-linearity the asymptotic slope increases in accordance with Eq. 18 while the time intercept (the "time delay") decreases in accordance with Eq. 29. As in the linear case the intrinsic diffusivity $\left(D_{0}\right)$ may be determined either from the time delay or from the limiting slope of the asymptote, provided that the upstream loading $\left(c_{1}\right.$ or $\left.\theta_{1}\right)$ is known. The directly measured quantities are:

$$
\left(\frac{\Delta Q}{\Delta t}\right)_{s s}=-\frac{D_{0} c_{1}}{\ell} \frac{\ln \left(1-\theta_{1}\right)}{\theta_{1}} \quad \text { and } t_{d}=\tau_{d}\left(\theta_{1}\right) \frac{\ell^{2}}{D_{0}}
$$




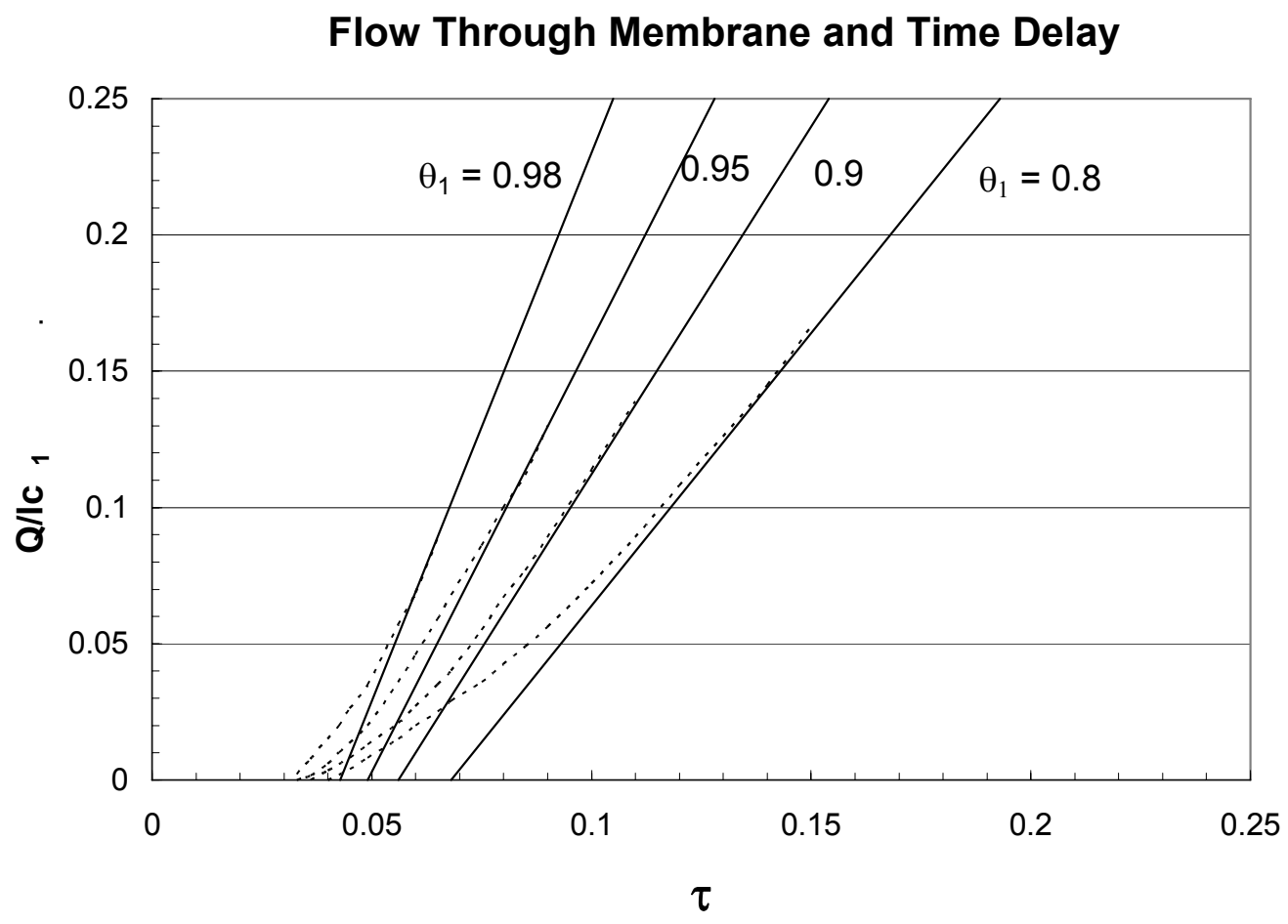

Fig. 4 Variation of flow through membrane $\left(Q / \ell c_{1}\right)$ with time $(\tau)$ for different values of $\theta_{1}$ showing the approach to the steady state asymptote defined by Eq. 30

so it is evident that, both the value of $D_{o}$ and the membrane thickness $(\ell)$ can be determined. Since the thickness of a zeolite membrane is often not amenable to direct measurement this is clearly advantageous. For a linear system the slope and time delay are given respectively by Eqs. 9 and 8 so the ratios of slopes and time delays for Langmuirian and linear systems, which correspond to the ratios $\left(D_{a p p} / D_{0}\right)$, are given by:

$$
\begin{aligned}
& \text { Slope: } \quad \frac{(\Delta Q / \Delta t) s s-\text { Langmuir }}{(\Delta Q / \Delta t) \text { ss - Linear }}=-\frac{\ln \left(1-\theta_{1}\right)}{\theta_{1}}=\left(\frac{D_{a p p}}{D_{0}}\right)_{\text {Slope }} \\
& \text { Intercept: } \quad \frac{\left(t_{d}\right) \text { linear }}{\left(t_{d}\right) \text { Langmuir }}=\frac{1}{6 \tau_{d}\left(\theta_{1}\right)}=\left(\frac{D_{\text {app }}}{D_{0}}\right)_{\text {Intercept }}
\end{aligned}
$$

These ratios are plotted as a function of $\theta_{1}$ in figure 5. For a given value of $\theta_{1}$ the slope and intercept ratios are similar (although not exactly equal) and converge at low and high loadings. Coincidence between the diffusivity values derived from the slope and intercept has been regarded as justification for the use of the simple linear model. However, the above analysis shows clearly that approximate coincidence (within experimental error) is to be expected between slope and intercept values even for highly non-linear systems. 


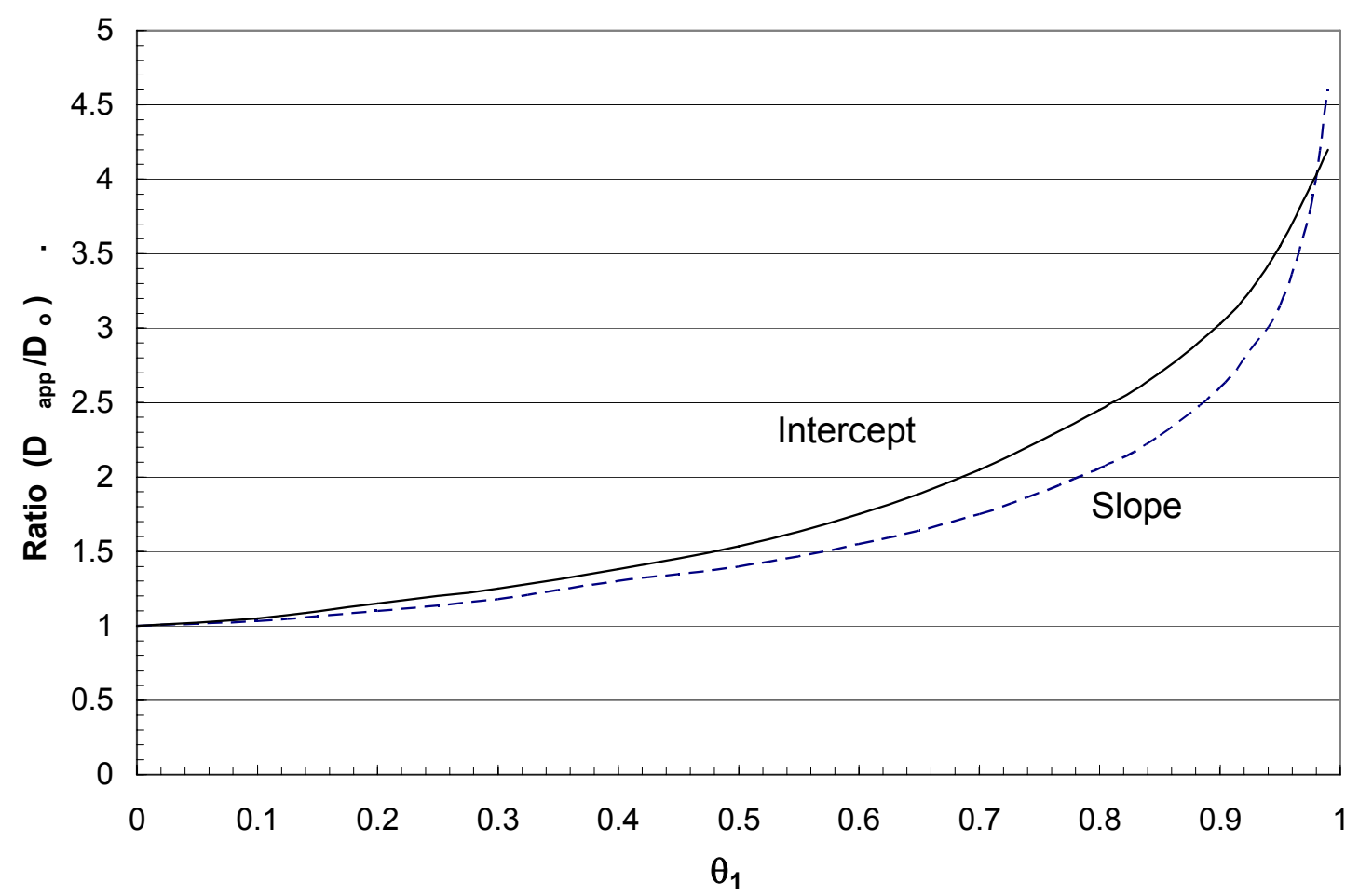

Fig. 5 Variation of correction factors $\left(D_{\text {app }} / D_{0}\right)$ with $\theta_{1}$ for slope and intercept values of diffusivity (Eqs. 31 and 32)

The limiting behavior as $\theta_{1} \rightarrow 1.0$ is of interest since this defines the behavior of a system with a highly favorable (rectangular) isotherm. It appears that in this limit $\tau_{\mathrm{d}}$ approaches a finite value of about 0.04 (see figure 2). If this is true then the intercept ratio, defined by Eq. 31 will reach a maximum value of about 4.2 at $\theta_{1}=1.0$ while the slope ratio will continue to increase indefinitely, approaching the limit $\theta_{1}=1.0$ asymptotically. This implies that, for highly favorable isotherms, the apparent diffusivity calculated from the slope will be substantially greater than that derived from the time delay. Such behavior has been observed experimentally in the work of Shah and Liou ${ }^{(4)}$. However, the validity of the simplified theoretical model becomes questionable in this limit and, as a result of the very strong concentration dependence of the diffusivity, numerical calculations also become inaccurate in this region so the possibility that the experimental confirmation of this behavior is fortuitous cannot be excluded.

\section{Analysis of Experimental Data}

Shah and Liou ${ }^{(4)}$ have presented an extensive set of permeation/diffusion measurements for aromatic hydrocarbons in a single crystal silicalite membraneoriented along the long (z) axis $(\ell=100 \mu \mathrm{m})$. The measurements were made mainly at $298 \mathrm{~K}$ at pressures ranging from 0.5 to 10 Torr. Under these conditions the equilibrium isotherms are highly non-linear, especially for $\mathrm{p}$ xylene for which $\theta_{1}>0.98$, yet the permeation data were interpreted in accordance with the classical linear model to yield apparent integral diffusivities. Intrinsic diffusivities were not 
Table 1

Variation of Breakthrough Time and Time Delay

\begin{tabular}{|l|l|l|l|l|l|l|l|}
\hline$\Theta_{1}$ & $\ell^{1} / \ell$ & $\mathrm{c}_{0} / \mathrm{c}_{1}$ & $\tau_{0}$ & $\tau_{\mathrm{d}}^{1}$ & $\tau_{\mathrm{d}}$ & $1 / 6 \tau_{\mathrm{d}}$ & $\left(\mathrm{D}_{\mathrm{app}} / \mathrm{D}_{0}\right)_{\text {slope }}$ \\
\hline & & & & & & & \\
\hline $\begin{array}{l}\text { Linear } \\
\left(\theta_{1}=0\right)\end{array}$ & 1.0 & - & 0.063 & 0.104 & 0.167 & 1.0 & 1.0 \\
\hline 0.5 & 0.722 & - & 0.051 & 0.107 & 0.107 & 1.56 & 1.38 \\
\hline 0.8 & 0.497 & 0.21 & 0.04 & 0.115 & 0.068 & 2.44 & 2.01 \\
\hline 0.9 & 0.39 & 0.15 & 0.036 & 0.132 & 0.056 & 3.0 & 2.55 \\
\hline 0.95 & 0.317 & 0.13 & 0.033 & 0.135 & 0.047 & 3.5 & 3.15 \\
\hline 0.98 & 0.26 & 0.095 & 0.032 & 0.143 & 0.042 & 4.05 & 4.0 \\
\hline 0.99 & 0.215 & 0.025 & 0.032 & 0.161 & 0.04 & 4.2 & 4.65 \\
\hline
\end{tabular}

$\ell^{1} / \ell$ is calculated from $\mathrm{Eq} .22$

$c_{0} / c_{1}$ and $\tau_{0}$ are from Fujita's solution

For $\theta_{1} \geq 0.8 f\left(X^{1}\right)$ is approximated by $\mathrm{e}^{-\mathrm{kX}^{1}}$ where $k=3.9$ and $\sum_{\mathrm{m}=0}^{\infty}\left[\mathrm{k}^{2}+(2 \mathrm{~m}+1)^{2} \pi^{2}\right]^{-1} \approx 0.061$.

For $\theta_{1}=0.5$ the exponential approximation is poor so $I(n)$ is evaluated numerically.

$\tau_{d}$ is calculated from $\mathrm{Eq} .29$ with $\tau_{d}^{1}$ from $\mathrm{Eq} .28$.

For the linear case $\left(\theta_{1} \rightarrow 0\right)$ values of $\tau_{0}, \tau_{d}^{1}$ and $\tau_{d}$ are calculated directly from Eq. 5 .

$1 / 6 \tau_{d}$ represents the ratio $\left(D_{a p p} / D_{0}\right)$ calculated from the time at delay (Eq. 32$)$.

derived. In figure 5 the "slope" and "intercept" diffusivities originally reported are compared with the corrected values, derived by application of Eqs. 31 and 32. The corrected diffusivities calculated from the non-linear model are smaller by factors of 4 to 5 , relative to the original integral values, and show much greater consistency between the "slope" and "time delay" values. The "slope" diffusivities derived from the linear model are greater than the "time delay" values by factors of 1.5 to 2.0. This result is in accordance with the predictions of the non-linear model for a highly favorable isotherm, as noted above.

Limited experimental data showing the temperature dependence of the diffusivity for $\mathrm{p}$ xylene at 2.17 Torr are also reported by Shah and Liou ${ }^{(4)}$. Since the pressure was maintained constant the loading will vary with temperature. The "time delay" values which do not depend on precise knowledge of the equilibrium loading are therefore considered to be more reliable. These data are plotted in Arrhenius form in figure 7 together with the corrected $\mathrm{D}_{0}$ values derived from the non-linear analysis assuming that at all temperatures $\theta_{1}>0.98$ so that $\tau_{d}$ is always close to its limiting value of 0.04. Also shown are experimental values of $D_{0}$ and $D$ obtained from ZLC, TZLC, and gravimetric measurements at higher temperatures. It is evident that the activation energies for both data sets are almost identical $(\sim 27 \mathrm{~kJ} / \mathrm{mole})$. The original membrane diffusivity values are approximately double the $\left(D_{0}\right)$ values extrapolated from the ZLC/gravimetric data whereas the "corrected" membrane values are smaller than the ZLC values by a similar factor and coincide with the extrapolation of the TZLC self-diffusivities. 


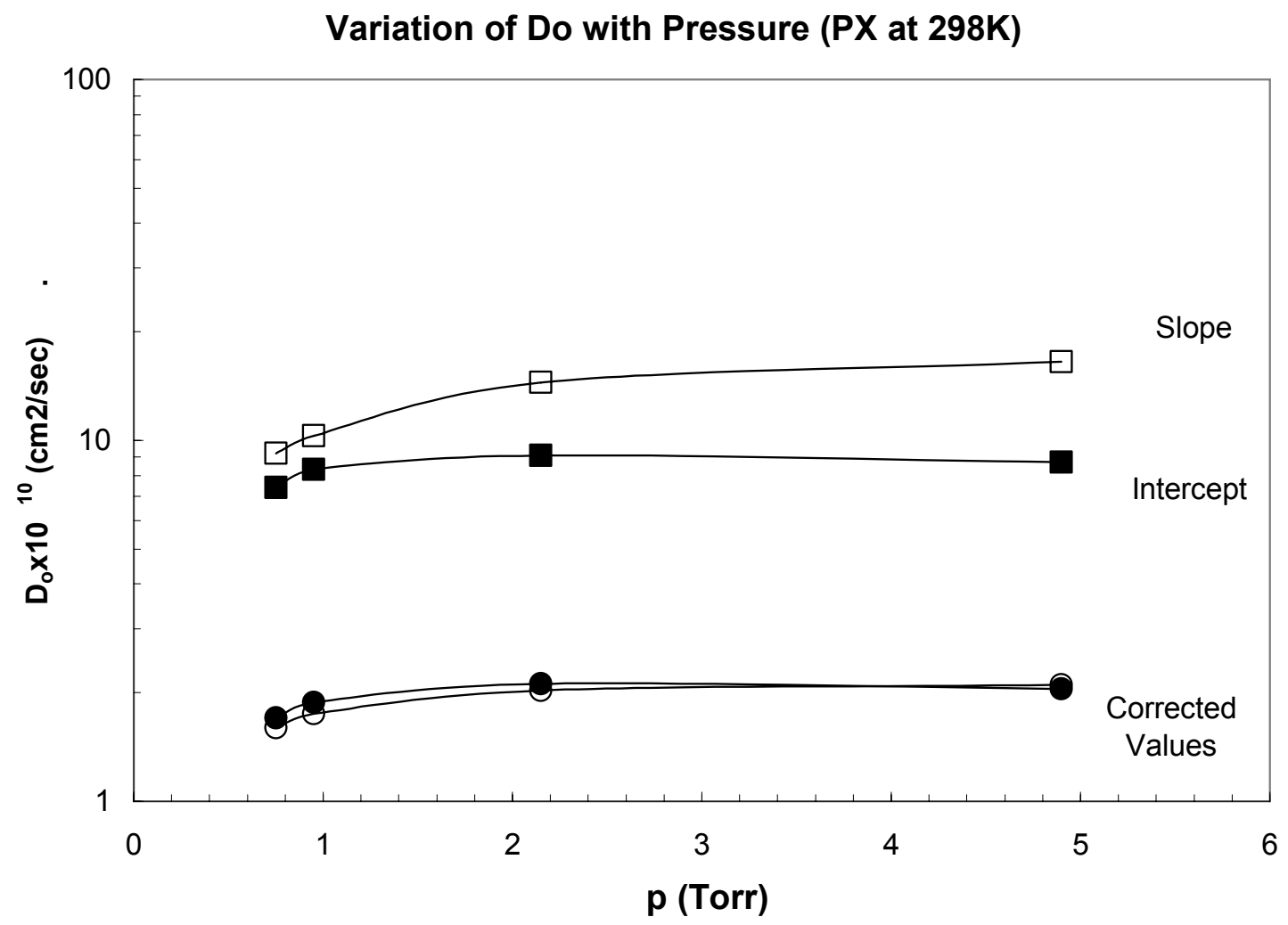

Fig.6 Variation of diffusivity with sorbate pressure for p-xylenes in a single crystal zeolite membrane at $298 \mathrm{~K}$ showing the comparison between "slope" and "time delay" values derived from the linear and non-linear models. Data of Shah and Liou $^{(4)}$

Diffusion in the direction of the long ( $\mathrm{z}$ ) axis of a silicalite crystal requires the diffusing molecule to jump successively between the straight and sinusoidal channel systems. The long $p$-xylene molecule can execute such jumps only with difficulty so the diffusivity in the $\mathrm{z}$ direction is smaller than that in the other directions and consequently smaller than the average value measured by ZLC or uptake measurements. Self-diffusion also requires the p-xylene molecules to pass between the two channel systems in order to pass one another so the coincidence between the self-diffusivity and the transport diffusivity in the z-direction appears physically reasonable. The "corrected" membrane values, which represent transport diffusion in the $\mathrm{z}$ direction, therefore appear to be consistent with the ZLC and gravimetric data.

\section{Conclusions}

The simple analytic model proposed here depends on the assumptions that the equilibrium isotherm is Langmuirian and the corrected diffusivity is independent of loading. These assumptions are at best approximations which become progressively less valid at high loadings. Nevertheless the model appears to provide a good representation of the experimentally observed behavior and leads to the following specific conclusions: 


\section{Temperature Dependence of $D_{o}$ for $p$-Xylene}

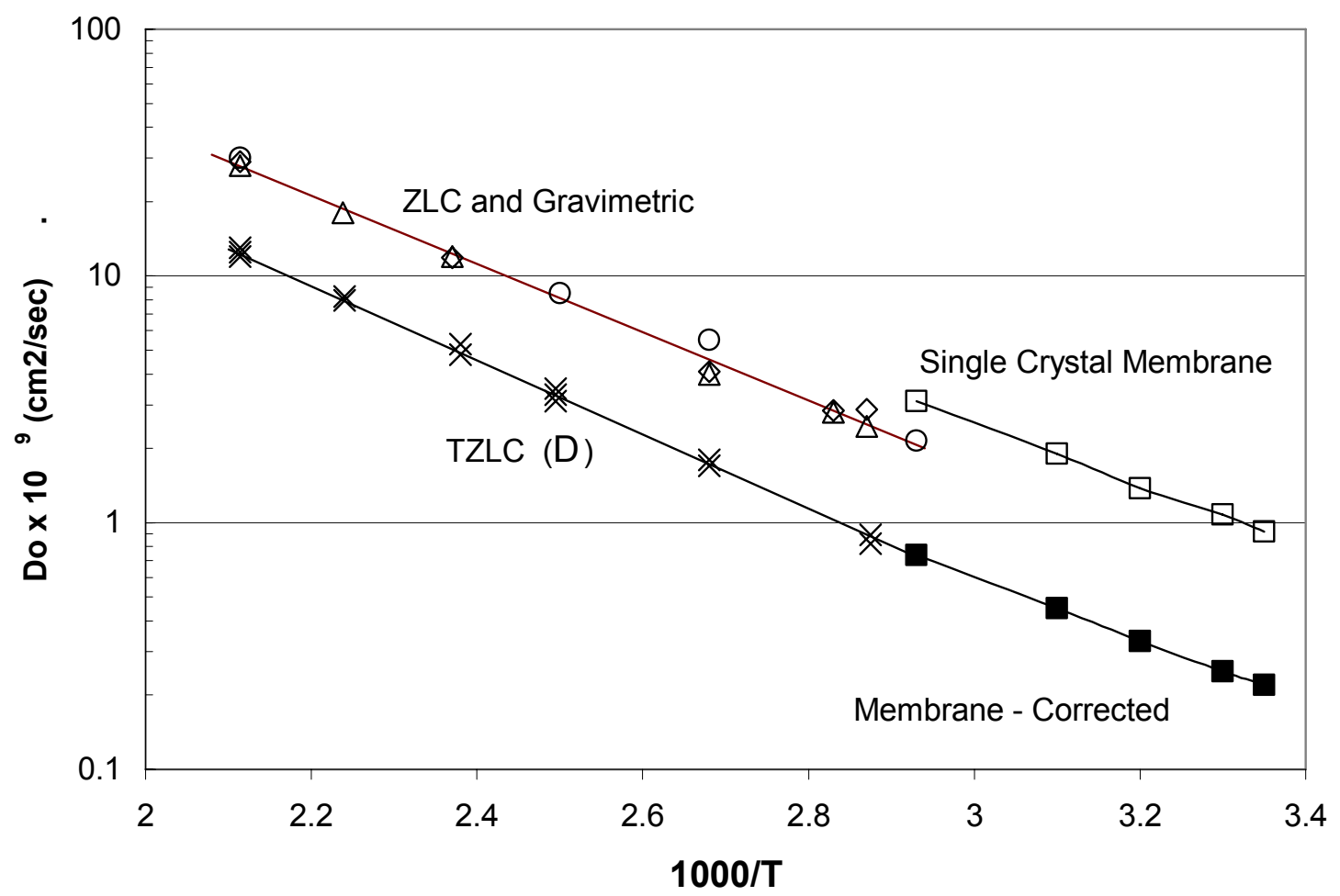

Fig. 7 Arrhenius plot showing temperature dependence of the limiting diffusivity $\left(\mathrm{D}_{0}\right)$ and tracer self-diffusivity (D) for p-xylene/silicalite and the comparison between ZLC, gravimetric and corrected single crystal membrane data. (Data are from refs. $4,7$ and 8$)$

1. The model provides a useful description of the transient behavior of a membrane under non-linear conditions and thus extends the time delay method of measuring diffusion to nonlinear systems.

2. Application of the classical linear model to a non-linear (Langmuirian) system will yield erroneously high apparent diffusivity values which depend on the loading level at the upstream face.

3. Even under non-linear conditions the diffusivity values derived from slope and time delay should be similar except at very high loadings $\left(\theta_{1} \rightarrow 1.0\right)$ under which conditions the slope values will be higher than the time delay values.

4. Application of the non-linear model to reported data for diffusion of p-xylenes in silicalite yields diffusivity values which are self consistent and are also consistent with the transport and self-diffusivity values derived from ZLC and uptake rate measurements. 
5. The observation that the limiting transport diffusivity $\left(D_{0}\right)$ in the z-direction (derived from the single crystal membrane measurements) coincides with the self-diffusivity (measured by TZLC) suggests that both these processes are controlled by the frequency with which the pxylene molecules can jump between the straight and sinusoidal channels. Transport diffusion in a silicalite crystal is clearly non-isotropic, as shown by the difference between the average value of $\mathrm{D}_{0}$ (ZLC) and the value of $D_{0}$ in the z-direction derived from the single crystal membrane measurement. The above model implies that self-diffusion should be isotropic and this conclusion could be tested experimentally by repeating the single crystal membrane measurements under tracer exchange conditions in a Wicke-Kallenbach system.

\section{Notation}

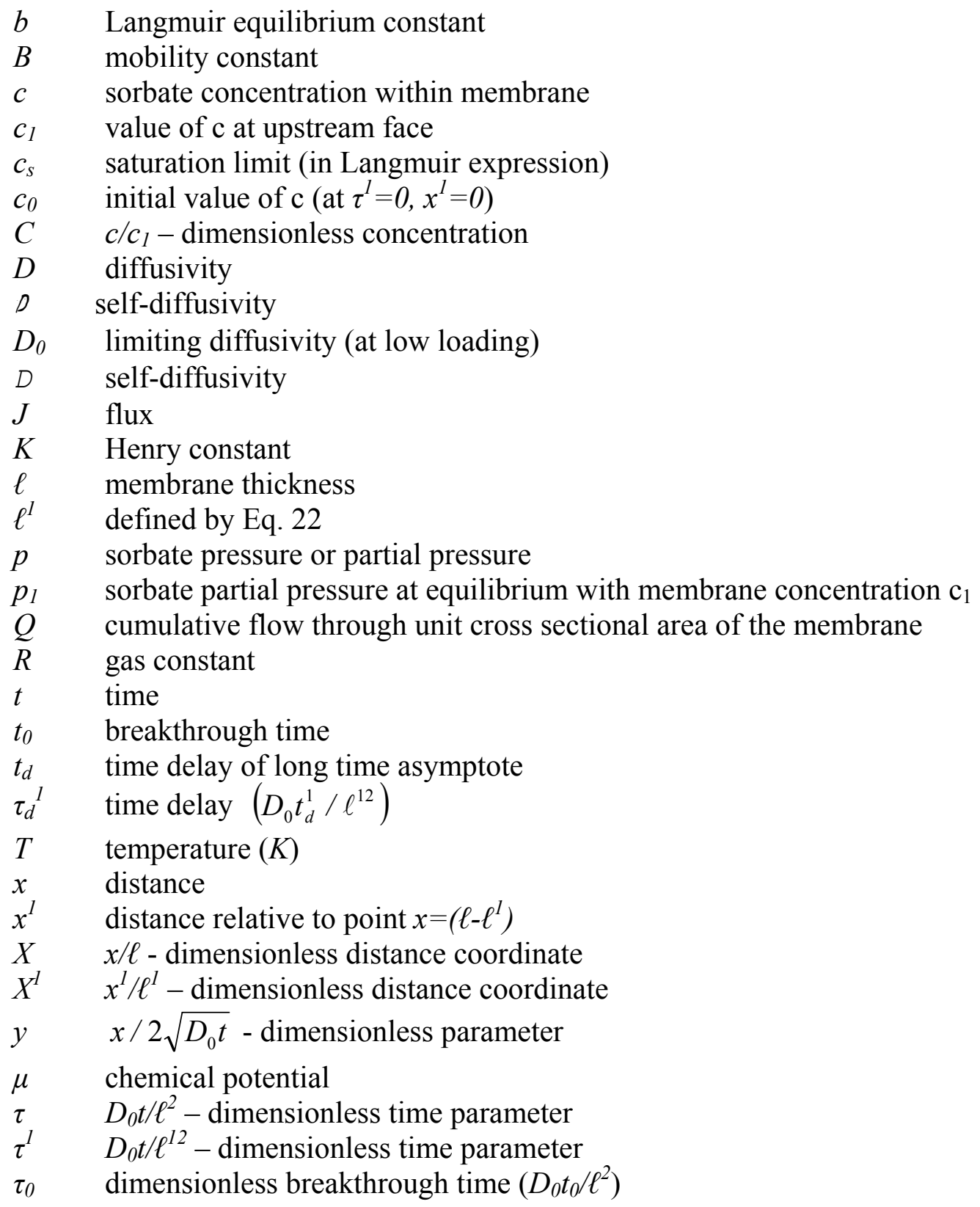


$\tau_{d} \quad$ time delay (or intercept of long time asymptote) $\left(D_{0} t_{d} / \ell^{2}\right)$

$\theta \quad c / c_{s}$ fractional loading

$\theta_{1} \quad c_{1} / c_{s}$ fractional loading at upstream face

\section{References}

1. J. Crank, The Mathematics of Diffusion Ch. IV, Oxford University Press, London (1956)

2. R.M. Barrer, Diffusion in and Through Solids p. 19, Cambridge University Press (1941)

3. D.T Hayhurst and A. Paravar, Zeolites 8, 27 (1988)

4. D.B. Shah and H.Y. Liou, Zeolites 14, 541 (1994)

5. A.Paravar and D.T.Hayhurst, in Proc. Sixth Internat. Zeolite Conf. (Reno, NV, 1983), pp122-127. A.Bisio and D.Olson eds. Butterworth, Guildford, U.K. (1984).

6. H. Fujita, Textile Research Journal 22, p.757 and 823 (1952)

7. D.M. Ruthven, M. Eic and E. Richard, Zeolites 11, 647 (1991)

8. S. Brandani, M. Jama and D.M. Ruthven, Microporous and Mesoporous Mats. 35-36, 283 (2000)

\section{Appendix}

Fujita's solution may be written in the following form:

$$
\begin{aligned}
& \theta_{1} C=1-e^{-2 I(\phi)} ; \quad \theta_{1}=1-e^{-2 I(1)} \\
& y \equiv \frac{X}{2 \sqrt{\tau}}=\frac{1}{\sqrt{2 \mu}}[f(\phi)-\phi] e^{I(\phi)} \\
& I(\phi)=\int_{0}^{\phi} \frac{d \phi}{f(\phi)} ; \quad f(\phi)=\sqrt{\phi^{2}-2 \mu \ell n \phi}
\end{aligned}
$$

These equations define $C(X, \tau)$ with $\phi, \mu$ as auxiliary parameters which are related to $\theta_{1}$ through the definitions of $f(\phi), I(\phi)$ and $I(1)$.

\section{Acknowledgement}

Financial support from the National Science Foundation (Grant CTS-0553861) is gratefully acknowledged. 